\title{
Article \\ Optimization and Coordination of Electric Vehicle Charging Process for Long-Distance Trips ${ }^{\dagger}$
}

\author{
Jean Hassler ${ }^{1,2,3}{ }^{(D}$, Zlatina Dimitrova ${ }^{2}$, Marc Petit ${ }^{1,3}$ and Philippe Dessante $1,3, * \mathbb{D}$ \\ 1 Laboratoire de Génie Electrique et Electronique de Paris, Sorbonne Université, CNRS, 75252 Paris, France; \\ jean.hassler@geeps.centralesupelec.fr (J.H.); marc.petit@centralesupelec.fr (M.P.) \\ 2 Groupe PSA, Route de Gisy, 78140 Vélizy-Villacoublay, France; zlatina.dimitrova@stellantis.com \\ 3 Laboratoire de Génie Electrique et Electronique de Paris, Université Paris-Saclay, CentraleSupélec, CNRS, \\ 91192 Gif-sur-Yvette, France \\ * Correspondence: Philippe.dessante@centralesupelec.fr; Tel.: +33-1-69-85-15-23 \\ + This paper is an extended version of our paper published in IOP Conference Series: Materials Science and \\ Engineering, Volume 1002, International Scientific Conference on Aeronautics, Automotive and Railway \\ Engineering and Technologies (BulTrans-2020), 10-13 September 2020, Sozopol, Bulgaria.
}

Citation: Hassler, J.; Dimitrova, Z.; Petit, M.; Dessante, P. Optimization and Coordination of Electric Vehicle Charging Process for Long-Distance Trips. Energies 2021, 14, 4054. https://doi.org/10.3390/ en14134054

Academic Editors: Nicu Bizon and Aldo Sorniotti

Received: 12 May 2021

Accepted: 25 June 2021

Published: 5 July 2021

Publisher's Note: MDPI stays neutral with regard to jurisdictional claims in published maps and institutional affiliations.

Copyright: (c) 2021 by the authors. Licensee MDPI, Basel, Switzerland. This article is an open access article distributed under the terms and conditions of the Creative Commons Attribution (CC BY) license (https:/ / creativecommons.org/licenses/by/ $4.0 /)$.

\begin{abstract}
Battery electric vehicles offer many advantages in terms of performance and zero-emission pollutants, but their limited range for long-distance trips compromises their large-scale market penetration. The problem of range can be solved with a dense network of fast-charging stations and an increase in embedded battery capacity. Simultaneously, improvements in high-power charging point units offer range gains of hundreds of kilometers in a mere $20 \mathrm{~min}$. One risk remains: The travel time depends on the availability of charging stations, which can drop during rush hours, due to long queues, or power grid constraints. These situations could significantly affect the user experience. In this paper, we presented an approach to coordinate EV charging station choices in the case of long-distance trips. This system relies on vehicle-to-infrastructure communications (V2X). The objective is to enhance the use of the infrastructure by improving the distribution of vehicles between the different charging stations, thus reducing waiting time. Our target is to build an efficient and easily deployable system. The performance of this system is compared to an uncoordinated situation and an offline optimization. We conducted a case study on a 550-km highway with heavy traffic. With this system, the results showed a $10 \%$ reduction in time spent in charging stations.
\end{abstract}

Keywords: electric vehicle; fast charge; simulation; optimization

\section{Introduction}

In the IPCC (Intergovernmental Panel on Climate Change) special report "Global warming of $1.5^{\circ} \mathrm{C}^{\prime \prime}$ [1], the first chapter highlights the consequences of global warming of more than $1.5^{\circ} \mathrm{C}$ and links it to greenhouse gases. The second chapter demonstrates the need to return to a zero-carbon footprint as soon as possible and estimates the remaining carbon credit for the temperature to exceed preindustrial temperature by no more than $1.5^{\circ} \mathrm{C}$. This emission-reduction requirement is to be applied in all emitting sectors, in particular in the transport sector [2].

Within this sector, we must consider several strategies: the optimization of existing solutions, as well as the use of alternative energy storage and propulsion systems. The promotion of battery electric vehicles, which do not emit any gas when driving (for a tank-to-wheel evaluation), appears promising. However, a broad approach is needed through life-cycle analysis and well-to-wheel studies. This type of study, presented in $[3,4]$, clearly shows the potential reduction in greenhouse gas emissions, while pointing to the battery production phase and the electricity production mix used, as having a substantial impact on the final balance. 
The advantage of EV (Electrical Vehicle) in terms of greenhouse gas emissions demands a further study of its acceptability and economic viability. First, EVs are adapted to city trips, with high efficiency, and lower noise and air pollution than ICE vehicles [5]. Major global cities are promoting its use by delineating low-emission areas. Vehicle-to-grid (V2G) technologies enable the use of the EV batteries for power grid services: Electric vehicle fleets can offer a frequency or voltage regulation service by extracting or supplying electrical power to the grid in a pilot-controlled way [6].

However, the main barrier to the large-scale deployment of EVs is the low energy capacity of batteries in comparison with the gasoline of ICE (Internal Combustion Engine) vehicles. Batteries remain expensive, heavy, and bulky (even if the mass and volume energy densities are increasing), resulting in high purchasing costs and low inherent autonomy for use over long distances. Fast charging, with powers between 50 and $150 \mathrm{~kW}$, provides a solution to compensate the lower energy capacity, thus enabling the growing number of vehicles. During these long trips, the autonomy depends mainly on the driving style (maximum speed, acceleration profile), the weather (outside temperature, wind), the road profile, and the traffic $[7,8]$. We decided not to consider these factors in this study, as we will see later that they can be integrated without changing the conclusions.

The deployment of charging infrastructure is hence the prerequisite for the spread of electric vehicles. A well-established charging network increases effective electric vehicle range, relieving range anxiety, and reducing inconvenience in the charging process. Csonka and Csiszár [9] centered their research question around charging station positions to facilitate long-distance travel while meeting local demands on both the existing stations and the forthcoming installations. They put forward multi-criteria weighted methods for both the national roads and the districts. A system approach revealed criteria that affect charging stations' popularity: demographic, economic, environmental, and transportationrelated attributes, and available services (points of interest). In [10], Funke et al. compared the utility of investing in fast-charging infrastructure or longer battery ranges, pointing out the cost efficiency of an infrastructure increase. In [11], Kosmanos et al. discussed on the potential interest of mobile charging stations, using vehicle-to-vehicle power transfer. As it is intended to city drive, we did not include it in our study.

In the present paper, we are motivated by the solutions that must be implemented to coordinate the choices of EV quick charge, especially for long-distance trips. Allowing electric vehicle drivers to avoid queues is potentially timesaving for users in addition to improve the usage of the charging infrastructure, consequently optimizing the required investments. However, the smart charging solutions must be designed to be easily implemented. Razo and Jacobsen, in [12], offered a model that guarantees the confidentiality of user data, in particular their vehicle condition and destination. It is based on reservations, transmitted by mobile networks, which allow charging stations to estimate waiting times and vehicles to choose stations based on these data. Gusrialdi et al., in [13], offered a solution that only requires vehicle-to-vehicle communications (V2V), including a cooperative exchange between vehicles, to determine who should stop at the next charging station. They based their work on Qin and Zhang [14], who showed that the optimal solution to this relaxed problem is achieved when the occupancy rate is constant, meaning when the vehicles are equally distributed at different charging points. Tan and Wang [15], on the other hand, used game theory tools to allow users to optimize travel costs and allow charging stations to maximize their revenues. They assumed hourly prices were freely set, and this resulted in a non-collaborative game. Overloading of the electricity grid was also considered. A cooperative approach to game theory was developed by Yang et al. [16], which allowed the convergence speed and robustness to be improved. However, these papers did not compare the performance of their solutions against optimal repartition. This approach did not allow for the evaluation of the room for improvement.

We proposed an approach with new communication scheme privacy which is computationally friendly. To compare it to the best solution, we use a differential evolution algorithm to find the offline optimal solution to this problem. 
This communication schema is meant to be easy to deploy and allows vehicles to be coordinated while reducing waiting times at charging stations during long-distance trips. The novelty of this article is the comparison of the interest between an achievable and affordable communication system, and the optimal solution.

In Section 2, we first present the methodology, the model, and the assumptions. We then detail the algorithm used for optimal-solution research in Section 3. Then, in Section 4, we describe the proposed communication scenario, with its structure and its calibration. Lastly, in Section 5, we apply this methodology to an EVs-flow simulation on a $550 \mathrm{~km}$ highway. This flow was composed of three types of EVs, with different battery sizes and charging powers of 50, 100, and $125 \mathrm{~kW}$. The highway has several quick-charging stations with different charging powers. This article was an extension of a previous article in a conference [17].

\section{Methodology}

\subsection{Model}

To compare users' recharging choice strategies, we developed in this paper a model to calculate the total time (trip, charging, and waiting) spent by a flow of electric vehicles on a highway. This function takes as its inputs, as shown in Figure 1:

- A highway layout, specified by its entrances, exits, and charging station characteristics (position, number of charging points, available power);

- A fleet of $N_{E V}$ vehicles, defined by their intrinsic attributes (battery capacity, maximum charging power, consumption) and their trip characteristics (start time, state of charge (SoC) at entrance, entrance, and exit points on the highway);

- The vehicle charging schedules, noted as $E_{j, n}$, the energy charged by EV $n$ at charging station $j$ (null if the vehicle does not stop).

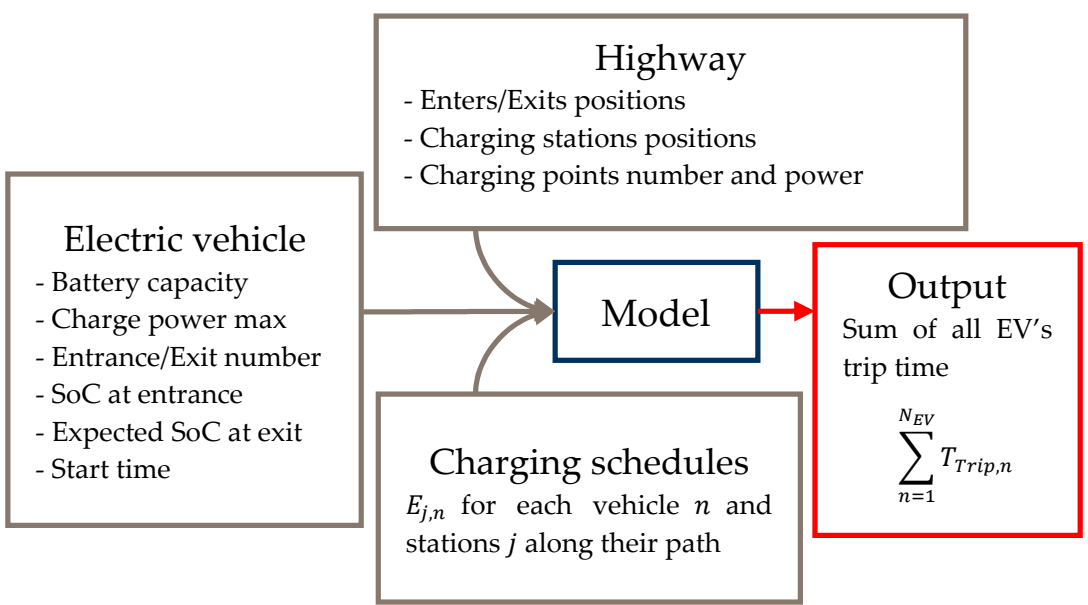

Figure 1. Structure of the simulation.

The function then computed travel times for all vehicles, considering queues at charging stations. Travel times, $T_{\text {Trip, } n}$, are composed of driving time $T_{\text {Driving, } n}$ and $N$ times in charging stations $T_{\text {Station } j, n}$, as described in Equation (1):

$$
\begin{gathered}
T_{\text {trip }, n}=T_{\text {Driving, } n}+\sum_{j=1}^{n} T_{\text {Stationj, } n} \\
T_{\text {Station } j, n}=T_{\text {Wait j,n }}+T_{\text {Charge j,n }}+T_{\text {Other } j, n}
\end{gathered}
$$

where $T_{\text {Wait } j, n}=$ waiting time for an available charging point when the station is full (in minutes), $T_{\text {Charge } j, n}=$ time required to store the intended amount of energy (in minutes), $T_{\text {Other } j, n}=$ constant, set here to $5 \mathrm{~min}$, representing time needed for all other operations: decelerating, accessing the station, launching the charging session, and leaving. 


\subsection{Assumptions}

We assumed in this article that the energy consumption was constant during the drive. This assumption led to the approximations of a few percent of the traveling and charging time.

We also assumed that all cars could charge at all charging stations. This hypothesis is not always correct: Several standards coexist depending on the region and some charging networks are proprietary and closed to other brands. However, an initiative is underway to converge the standards [18]. Further, the following work may apply to each of the CS and EV sets that are compatible with each other.

\subsection{Charging Power}

Figure 2 shows the exchanges of energy and information between an electric vehicle and a quick-charging point. The charging point performs the AC/DC conversion, and a DC cable transfers the power to the battery. The battery management system (BMS) monitors the battery status and sends a voltage and current setpoint to the charging point.

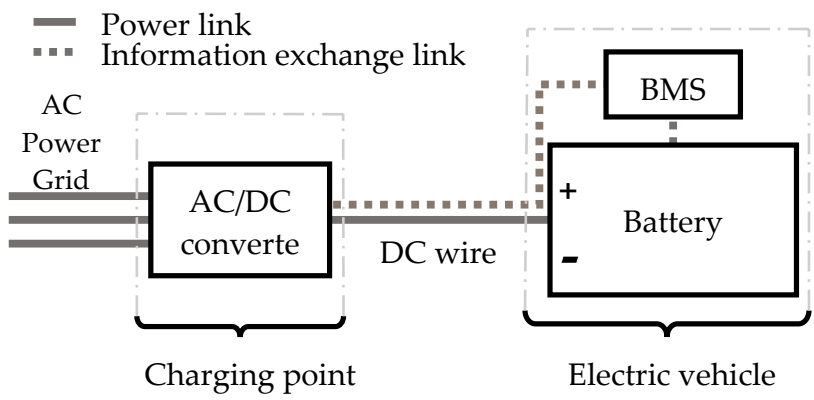

Figure 2. Information exchange and power flow chart between fast charging point and electric vehicle.

Effective charging power depends on the following parameters:

- The power that can be supplied by the network, which can become limiting when one network connection serves several terminals. Later in the article, we assumed that this portion was not narrowing;

- Maximum power of the charging station, depending on its design;

- Batteries' maximum charging power, which is detailed afterward.

Batteries' charging power depends mainly on its design, temperature, state of charge, and state of health.

We used the approach developed in [19]. This model furnishes the charging power as a function of the maximum charging power and the SoC. This charging power model is applicable for SoC between 0 to $80 \%$. For all cars, the charging power, when the SoC is $0 \%$, is equal to the maximum charging power. It then slowly and linearly decreases when the $\mathrm{SoC}$ increases. The slope depends on the vehicle type. Figure 3 shows the recharge powers as a function of the $\mathrm{SoC}$ for the three vehicles considered in this article: sedan, luxury, and urban. We also assumed that users recharged only up to $80 \%$ of SoC (power was drooping above this threshold) $\left(S_{0} C_{\max , n}=80 \%\right)$. 


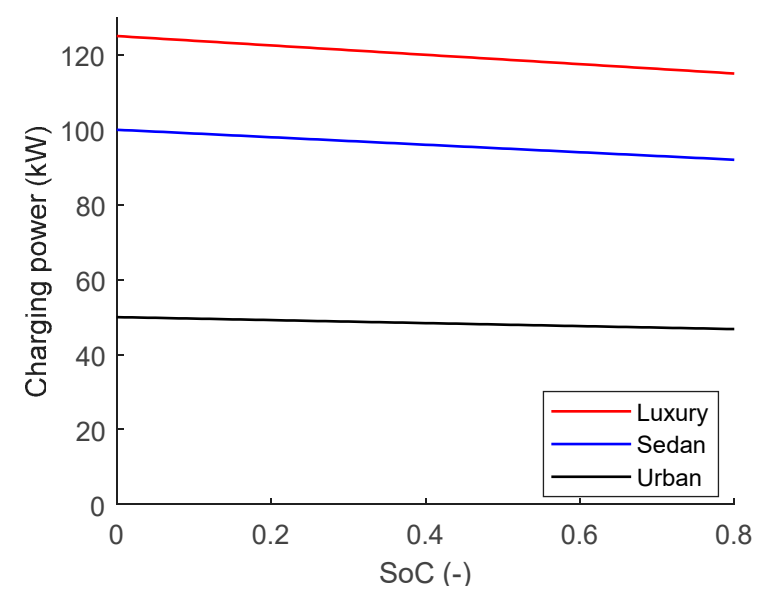

Figure 3. Charging power during quick charge for three types of vehicles.

\subsection{Model Application}

In the rest of this paper, we used this model in the following manner: Considering the characteristics of the motorway and the vehicle fleet of the day as fixed, we looked for charging schedules $\left(E_{j, n}\right)$ that minimized the sum of all travel times. We searched for these schedules with three algorithms:

(1) Without coordination: This was the reference case, for which there was no communication. It was the upper bound of the problem;

(2) Overall optimization: This was an offline search for the optimal solution. The algorithm considered all the journeys over a day. It then looked for the best distribution of vehicles between the different stations. This solution constituted a lower bound to the problem, for development purposes, and was not meant to be deployed;

(3) Coordination: It was a proposal for a communication structure between vehicles and stations. It was an online and deployable algorithm. Trips were known by the drivers but not by the system (charging stations).

Sections 3 and 4 present algorithms (2) and (3), respectively.

\section{Optimal Solution}

\subsection{Problem Characterization}

In this section, we introduce a method to find the best charging distribution over a day, which minimizes the sum of the fleet total trip time. For a given highway and fleet, we introduced the function $f$ as:

$$
f(\{E\})=\sum_{n=1}^{N_{E V}} T_{T r i p, n}
$$

with $\{E\}$ a set of charging plans $\left(E_{n, j}\right)$.

Then, we summed up the optimization definition by the minimization of this function under the constraints of SoC:

$$
\begin{gathered}
\min _{\{E\}} f(\{E\}) \\
\text { u.c. } \forall t, \forall n, \operatorname{SoC}_{\min , n} \leq \operatorname{SoC}_{n}(t) \\
\forall n, \operatorname{SoC}_{n}(t) \leq \operatorname{SoC}_{\max , n} \text { after recharge } \\
\forall n, S_{0} C_{\text {exit }, n} \leq \operatorname{SoC}_{\text {exit required }, n}
\end{gathered}
$$

$S_{0} C_{n}(t)$, So $C_{\min , n}$, and $S o C_{\max , n}$ respectively, represent the state of charge of vehicle $n$ at time $t$, its minimum and its maximum values. $S o C_{\text {exit }, n}$ and $S o C_{\text {exit required, } n}$ represent the SoC respectively at the highway exit obtained and required.

The optimal solution is the one that offers the best tradeoff in minimizing: 
- The number of stops, as it saves $T_{\text {other }}$;

- Waiting times, by finding the best distributions of EV between all charging stations;

- Charging times, by charging at the higher charging power and only the required energy.

\subsection{Resolution}

To solve this multiple-variable problem, we chose to use a differential evolution algorithm. This kind of algorithm uses a population of solutions and repeats a process of mutation, crossover, and selection [20]. At each iteration, each element incurs a mutation that is considered only if it permits to improve the solution. The mutations used are various classical vectorial combinations of randomly selected elements. However, this algorithm is not efficient in minimizing the number of EV stops. We therefore developed two other mutations that helped to test solutions with fewer stops. Figure 4 shows the mutations principle. The first one, mutation Move, transfers the full charge from one station to another on the vehicle route, whether it intended to stop or not. The second one, Distribute, spreads the charge from one station to other random stations where this EV already intended to stop. Through tests, we found that they do, indeed, accelerate the speed of convergence.

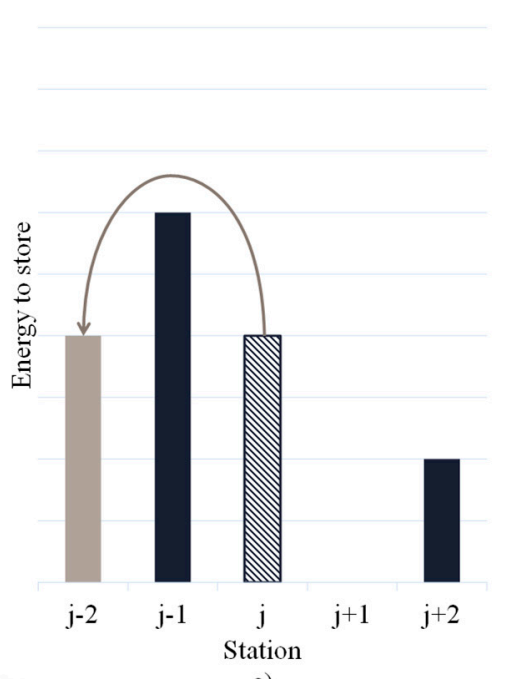

a)

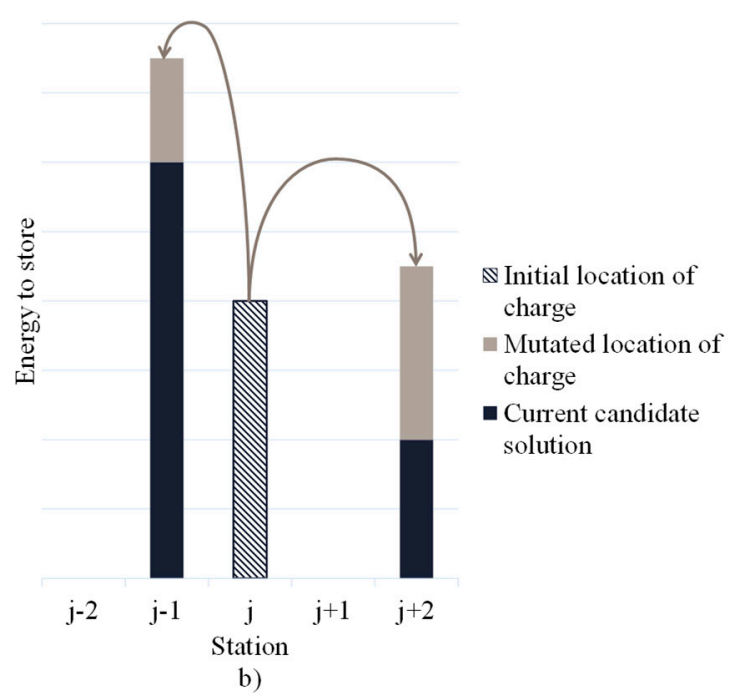

Figure 4. Proposed mutations (a) Move, (b) Distribute from an initial solution with scheduled stops at charging station $\mathrm{j}-1, \mathrm{j}$, and $\mathrm{j}+2$.

This methodology permitted us to obtain what we assumed to be the best-found solution for this offline problem as there was no certainty that this algorithm converges to the global minimum (we tested its convergence on a limited set of vehicles and charging station). But this algorithm took time to find the solution, as complexity increased exponentially with the number of vehicles (15 min on a 16 cores processor with $64 \mathrm{Go}$ of ram for $100 \mathrm{EVs}$ ) and was not privately friendly, as the centralized system where the computation is done has to know the travel path and the SoC of each vehicle. We propose a new approach to address these issues in Section 4, and we used this best-found solution to compare its performances.

\section{EV-CS Communication Scenario}

\subsection{Communication Issues}

In this section, we propose a coordination system relying on V2I communication. The primary constraint was that the deployment of this system had to be feasible in practice. It had several impacts on the architecture.

First, such a system relies on wireless connections between vehicles, charging stations, and a central server. These connections are based on mobile networks, the former technology operating for long distances, the latter for near communication (less than a few 
kilometers). The risk of dead zones and the latency of these communications demands that we set apart solutions requiring continuous or rapid exchanges.

Second, computing power must remain low to enable a large-scale deployment of the solution. Thus, a distributed system, without a centralized server, was chosen. Indeed, a centralized server gathering the requests of all users would allow for finding optimal solutions (Section 3). But its implementation, reliability, and required computing power could be a significant hurdle.

Third, users can be reluctant to share personal data, such as destination, average speed, or state of charge of the vehicle, for privacy and security reasons. Finally, constraints of robustness and cohabitation with other systems must also be considered.

Figure 5 shows the adopted architecture: communication only occurs between EV and CS (V2I: vehicle-to-infrastructure).

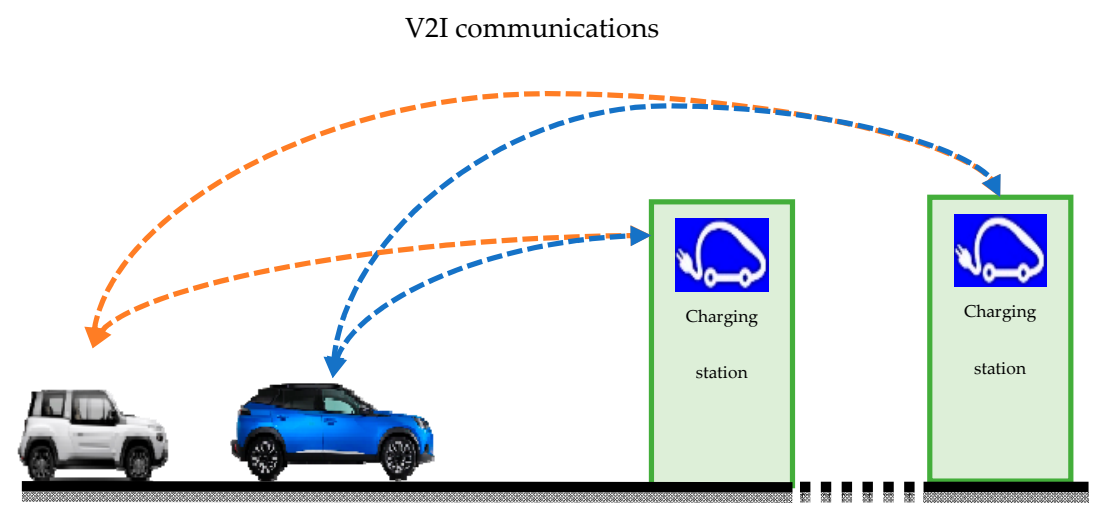

Figure 5. Proposed communication architecture.

\subsection{Proposed Coordination Schema}

Considering all the constraints mentioned in the Section 3.1, Figure 6 describes the original communication schema that was proposed. Communications between EVs and charging stations (CS) use mobile networks. This process, from the vehicle point of view, followed six steps:

1. The EV calculated several possible combinations of stops, with different stations and amounts of energy to be stored to guarantee to reach its destination. This computation was done on the vehicle calculator resources. Details of calculations are given in Section 4.3;

2. The EV requested the expected waiting time at charging stations at a specific arrival time, for all combinations;

3. CS responded to this request due to an updated arrivals list;

4. The EV could now choose the quickest charging plan, and it then sent a notification to the selected stations, including a given arrival time with an estimated charging duration;

5. The EV removed solutions that did not correspond to what it already did. For example, if it had already passed by station $j$ without stopping, it could remove solutions where $E_{j, n} \neq 0$

6. The vehicle repeated the cycle composed of the steps 2 to 5 , every period $T$.

The vehicle first ran this cycle when starting the trip, between the start and the highway entrance: allowing an anticipation of the schedule. It then refreshed according to period $T$. We noted $T_{a d v}$ the time between the beginning of the trip and the moment when the vehicle entered the highway. It had the same value for each vehicle. This system thus allowed each user to choose their best path according to the choice of others. This choice was built iteratively. 


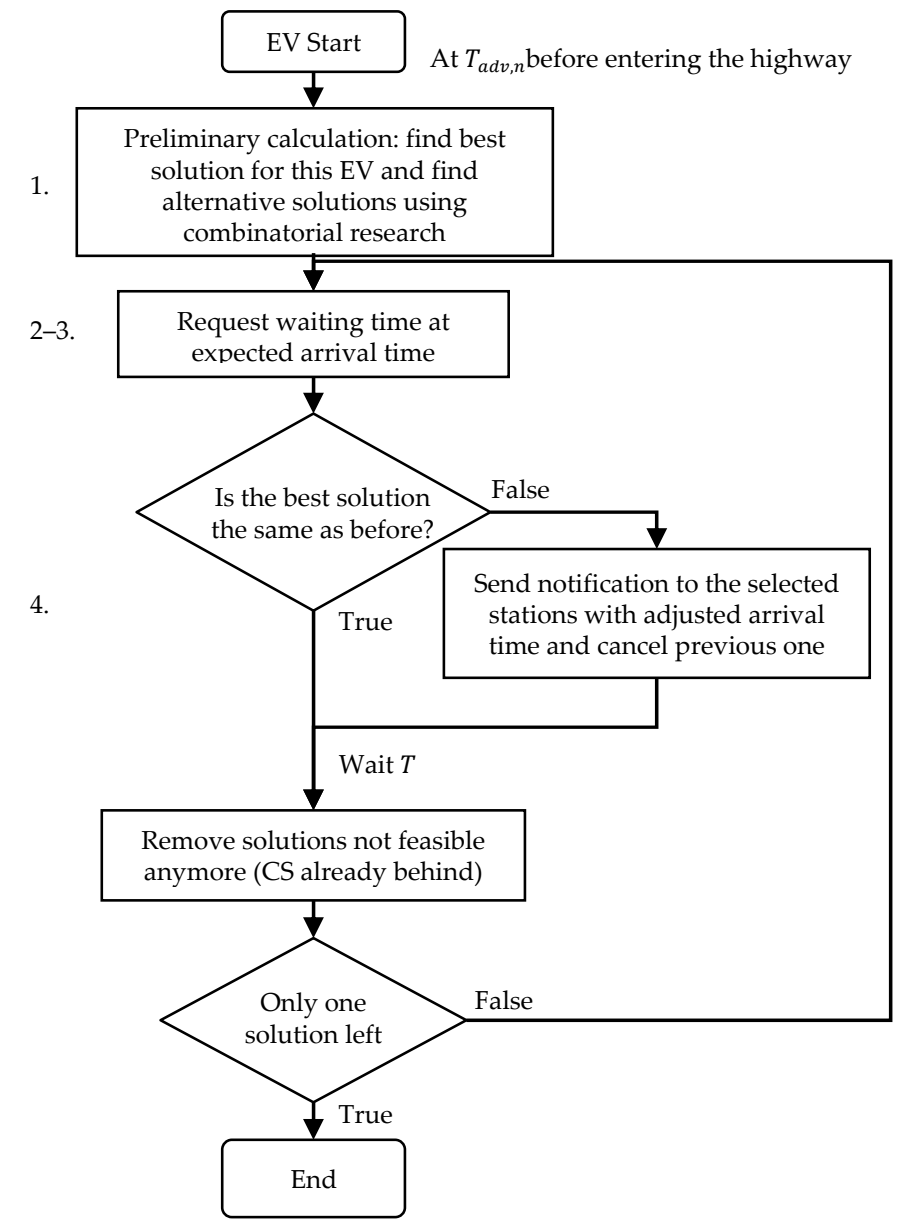

Figure 6. Communication flow chart from the vehicle point of view.

Therefore, three types of messages were exchanged:

- Waiting time requests sent at each period T;

- Expected arrival notification sent (from EV to CS) on the first cycle and whenever a faster route was found. It contained arrival time and charging duration;

- In the same way, notification of cancelation to CS that were no longer part of the schedule.

From the station point of view, the system was composed of a device that had to receive requests of waiting time estimation and notification of recharge, to then compute scheduled waiting times. This process followed these steps:

1. The station received arrival times and charge times of EVs planning to stop. It therefore calculated charging point usage and potential queuing;

2. In the event of a change on the EV side (change of plan in favor of another CS, slow down on the road, longer time spent at another station ... ), the EV notified the CS at the next $\mathrm{T}$ period, allowing it to update its list;

3. If this change affects the waiting time for other EVs, they would be notified at the next T-period, allowing them to change their plan if necessary.

It is worth noting that we assumed an information system, not a reservation system. Thus, the rule was first come, first served. As a result, a vehicle joining the highway in the middle, may arrive at a station before others who had planned to go there and increase their waiting time. This may result in a change of plan for these vehicles and others in a cascade. This risk of increased changes is dealt with in Section 4.4 when choosing the parameters $T$ and $T_{a d v}$. If the reservation is considered, it leads to a decrease of flexibility as when a vehicle takes a charging slot there is no opportunity for it to change later in time. 


\subsection{Charging Plans Research}

On the vehicle side, the search for the different charging plans, $\{E\}$, is carried out according to the following process:

- With a logic of recharging at the last reachable station, we found the first solution;

- We found the solutions that require the same number of stops as the first solution through a combinatorial search (or one more if the initial number of stops is one or two);

- These solutions were selected if they did not induce a trip $10 \%$ longer than the fastest route, to minimize the number of considered solutions.

The complexity of this calculation can significantly increase with the number of recharges and CS on the road. However, as these two numbers remain limited during an EV trip, this is not a limiting factor (computation time is always less than a minute). Moreover, since each EV performs its own calculation, the number of vehicles on the road does not impact the overall complexity.

Factors affecting range or speed, such as traffic or weather, could also be integrated into the system at this level. This would enable real-time scheduling adjustments to be made.

\subsection{Technical Feasibility}

The proposed coordination system can be easily implemented. First, the required computing power is low. On the vehicle side, the complexity of the calculation is of the same order of magnitude as the route search. It is, therefore, possible to use the same computer resources as those of the navigation system. On the station side, the amount of calculation to be performed depends on the number of vehicles on the road. However, as the computational complexity is low, they can be performed by an affordable computer system.

Secondly, it is a distributed system. Thus, it can be deployed over vast territories and vehicle fleets, without the risk of saturating a central server.

Moreover, this solution does not require sharing personal data such as localization, destination, state of charge of the battery, or driving speed.

Further, this system requires frequent exchanges of data between EVs and CSs at each time step $T$. However, the volume of this data (containing mainly timetables) is minimal compared with the use of mobile networks for other applications, such as media streaming. For a value of $T$ of a few minutes, the system should not overload the communication networks.

\section{Application}

5.1. Case Study

The generated data represent the situation of a highway in a day with 100 electric vehicles. The road is one way, 11 entrances and exits, and is $553 \mathrm{~km}$ long. It includes six charging stations, each with three charging points of 50, 100, or $125 \mathrm{~kW}$ maximum available power. Figure 7 displays its implantation. There are three types of vehicles in this simulation: urban, sedan, and luxury. Table 1 gives their respective characteristics. The consumption data come from the car manufacturers.

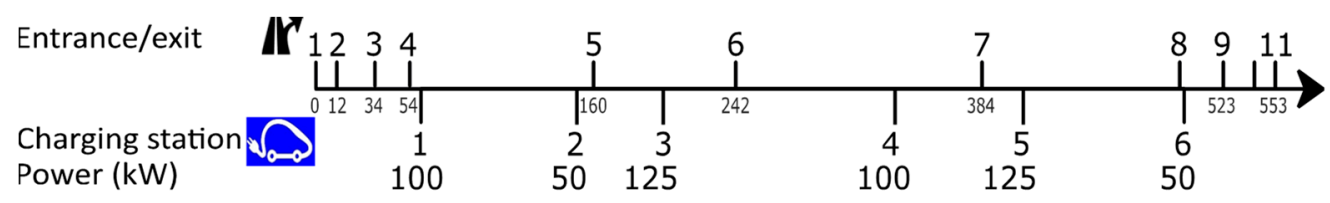

Figure 7. Highway characteristics. 
Table 1. Studied vehicles.

\begin{tabular}{cccc}
\hline Car Type & Urban & Sedan & Luxury \\
\hline Battery $(\mathrm{kW} . \mathrm{h})$ & 50 & 60 & 100 \\
Maximum charging power $(\mathrm{kW})$ & 50 & 100 & 125 \\
Consumption $(\mathrm{kW} . \mathrm{h} / \mathrm{km})$ & 0.15 & 0.18 & 0.18 \\
Driving Speed $(\mathrm{km} / \mathrm{h})$ & 110 & 130 & 130 \\
Frequency of occurrence & 0.3 & 0.6 & 0.1 \\
\hline
\end{tabular}

We based our daily highway flow modeling on the data found in [21]. Figure 8 shows the average daily flow of vehicles entering the French A6 motorway in the Ile-de-France region in the year 2017. These were the cumulative data of highway entrances equipped with inductive counting loops (blue curve). It indicated an almost constant flow between 8 a.m. to 9 p.m. We also noticed that the night's traffic was more than three times lower than during the day. As we wanted to study crowded situations, we focused only on the daytime scenario. Therefore, we used the simplified data shown in orange on the figure. We chose vehicles' entry and exit randomly, following a uniform distribution while imposing a minimum travel distance of $250 \mathrm{~km}$.

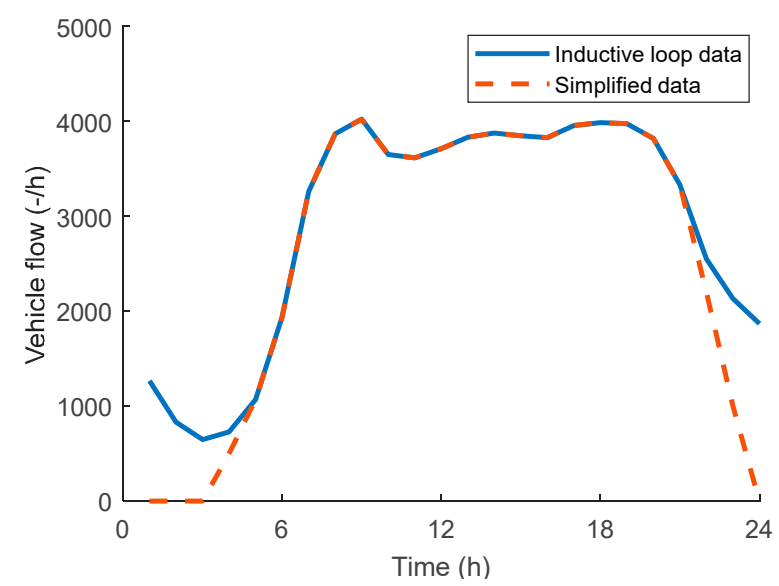

Figure 8. Average daily incoming vehicle flow.

We also used a uniform distribution for the SoC at departure, in the $50-100 \%$ range. $\mathrm{SoC}$ at arrival (when the car exits the highway) is required to be above $30 \%$, and $\mathrm{SoC}$ when entering a charging station to be above $15 \%$. EVs are charged to a maximum of $80 \%$ or below. Table 2 lists the parameters selection method.

Table 2. Parameter selection.

\begin{tabular}{cc}
\hline Parameter & Value \\
\hline Departure time & Figure 8 simplified data distribution \\
So $C_{\text {entrance }}$ & Uniform distribution $[50 ; 100] \%$ \\
So $C_{\text {exit required }}$ & $30 \%$ \\
So $C_{\text {Max }}$ & $80 \%$ \\
So $C_{\text {Min }}$ & $15 \%$ \\
Entrance & Uniform distribution $[1 ; 6]$ \\
Exit & Uniform distribution $\left[E_{X 1} ; 11\right]$, with $E_{X 1}$ first exit more than $250 \mathrm{~km}$ \\
& away from the chosen entrance \\
\hline
\end{tabular}

\subsection{Data Presentation: Trips Characteristics}

In this section, we do not consider waiting times at CSs to illustrate only the driver's needs. The following figures present the characteristics of the EV trips generated by the assumptions presented in Section 4.1. Figure 9 shows two histograms: the distribution 
of trip distances and trip times, considering recharge times. It shows the diversity of the routes studied and the fact that they are mainly between 400 and $550 \mathrm{~km}$.
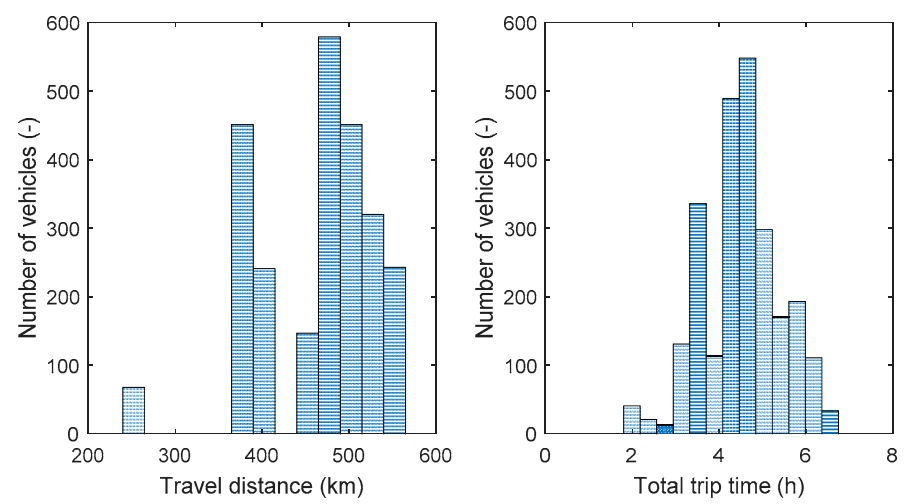

Figure 9. Generated vehicle trip characteristics.

\subsection{Preliminary Case Study}

We performed this first simulation with 100 vehicles in one day. It represents $1 \%$ of a long-distance highway traffic in France [22]. We compared three strategies:

- Without coordination: EV drivers have no information about choices made by other EVs. Each driver optimizes their charging schedules individually to minimize their charging time. This strategy reproduces the choices that a driver can make, with only knowledge of charging stations position and power.

- Coordination: Strategy defined in Section 4, we supposed that all drivers use the system. We set the period $T$ and $T_{a d v}$ at $10 \mathrm{~min}$.

- Global optimization: (Section 3) Situation in which all information is centralized and choices are made in optimally, considering waiting times when making choices. This strategy led to the best-found solution.

Figure 10 shows the average waiting time at the different stations for the three situations. The communication, and even more so the global optimization, can reduce average waiting times. This improvement was made possible by a better distribution of vehicles between stations. We noted that the decrease in waiting time at station 3 , the busiest station, did not induce a waiting-time increase at the other stations. The charging infrastructure was, therefore, better used.

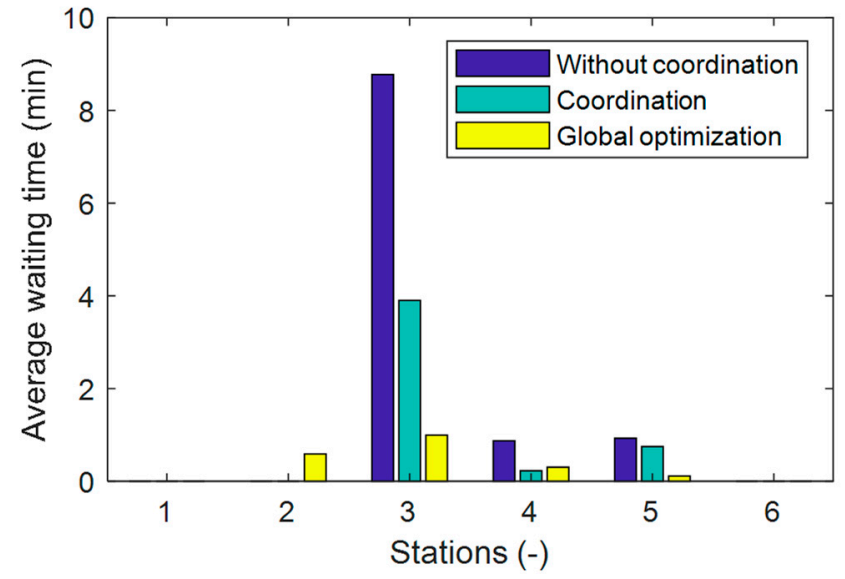

Figure 10. Average waiting time comparison. 


\subsection{Parametric Analysis}

The coordination system had two parameters: the refresh period $T$, and the anticipation time $T_{a d v}$. Before analyzing more of the results, we evaluated their impact on three outcomes that characterized the operation of the communication system:

- The average waiting time in CSs;

- The average charging time;

- $\quad N_{c h}$, the average number of times a vehicle changed its reservations after finding a combination of recharges that reduce trip time.

The last parameter reflects the system's complexity and the need for communication: Each change can lead to modifications in waiting times for other users and other cascading changes.

To assess this impact, we modeled the use of this communication scheme on a highway with flows of 80 and 150 vehicles per day. We simulated the behavior over 1000 generated days, following the case study presented in Section 4.1. This number of draws allowed the results to converge to a stable solution.

Figure 11 shows the variation of $N_{c h}$, in color scale, for $T$ values in $\{3,5,10,15,20\}$ minutes and $T_{a d v}$ ranging from 0 to $40 \mathrm{~min}$. It shows the progressive increase in $N_{c h}$ when $T$ decreases and when $T_{a d v}$ increases. The case where the number of changes is minimal is, therefore, for $\mathrm{T}$ being $20 \mathrm{~min}$ and $T_{a d v}$ being $0 \mathrm{~min}$. In this configuration, there was less than one change per vehicle on average during their trip. This means that connection needs were low compared to other situations. We also observed an increase in $N_{c h}$ by a factor of more than seven between the simplest configurations and those with a short update period and a large $T_{a d v}$.

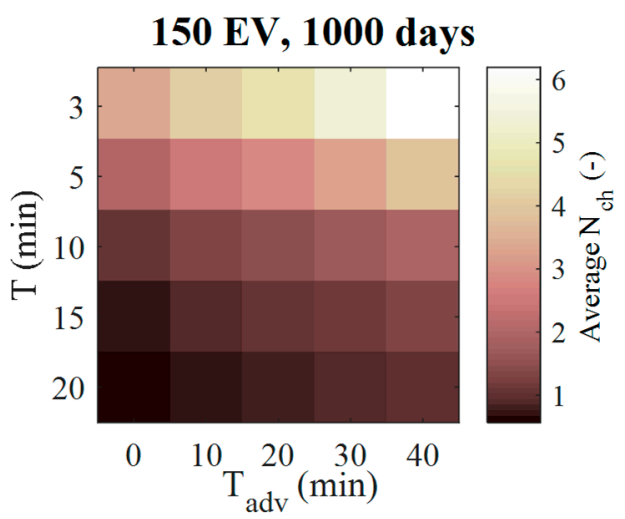

Figure 11. Average number of booking changes, with $150 \mathrm{EV}$.

Figure 12 shows the evolution of the average waiting time, according to the variables above, for 80 and $150 \mathrm{EVs}$. These numbers of vehicles represent various use situations of the studied infrastructure. It shows the impact of $T$ and $T_{a d v}$ on $T_{\text {wait }}$ : The average waiting time decreased as the $T$ period decreased, and as the $T_{a d v}$ time interval increased. The variation interval, of the order of $0.6 \mathrm{~min}$ for $80 \mathrm{EV}$ and $2 \mathrm{~min}$ for $150 \mathrm{EV}$, represented a $4 \%$ variation.

This study, therefore, revealed that the choice of $T$ and $T_{a d v}$ made it possible to optimize the communication algorithm's behavior by reducing the average waiting time of users. However, since the decrease in $T_{\text {wait }}$ by decreasing $T$ and increasing $T_{a d v}$ was of small amplitude, and the increase in complexity, shown by $N_{c h}$, was significant, we subsequently decided to set these parameters to an intermediate value offering a good compromise: $5 \mathrm{~min}$ for $T$ and $20 \mathrm{~min}$ for $T_{a d v}$. Indeed, this set of parameters $(5 ; 20)$, compared to $T$ being $3 \mathrm{~min}$ and $T_{\text {adv }}$ being $40 \mathrm{~min}$, induced a $1 \%$ increase of $T_{\text {wait }}$ while dividing the number of changes by a factor of two. Those parameters $(5 ; 20)$ induced an average of less than three reservation changes, as seen in Figure 11, which seemed technically feasible and not too troubling for the EV user. 


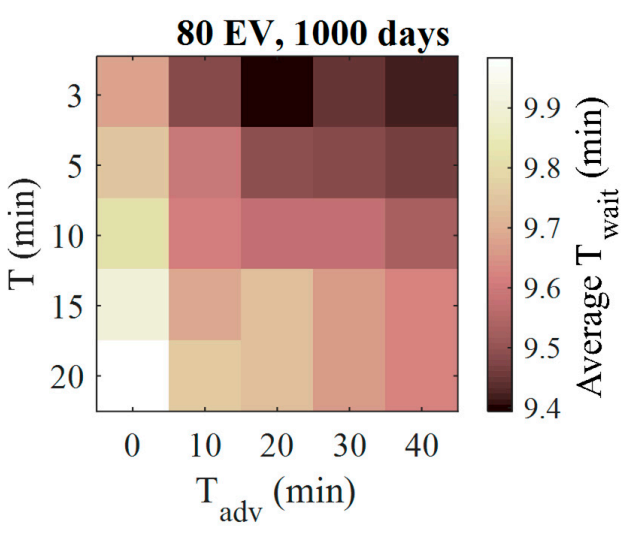

(a)

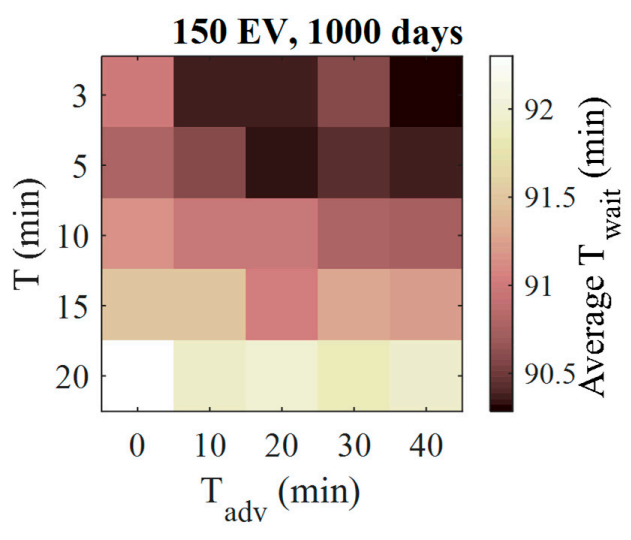

(b)

Figure 12. Evolution of average $T_{\text {wait }}$ as a function of $\mathrm{T}$ and $T_{\text {adv }}$ for (a) 80 and (b) $150 \mathrm{EV}$.

\subsection{Comparison}

We performed the simulations with 100 vehicles a day and averaged them over 15 days. To qualify a situation as satisfactory for users, we defined an arbitrary quality criterion. We considered the infrastructure as sufficient if it met the following thresholds:

- $90 \%$ of EVs entering a charging station had less than 5 min to wait;

- All waiting times were less than $30 \mathrm{~min}$.

The following figures show the results of the comparison between the three strategies. Figure 13 shows the cumulative distributions function of waiting times at the different stations for the three strategies, the dotted line representing the quality criterion defined above. The communication, and even more so the global optimization, could reduce waiting times for all drivers entering charging stations. Thus, for station 3 , the busiest, there was a decrease in the maximum waiting time from 74 to 40 and then $20 \mathrm{~min}$ (respectively for the three strategies) and an increase in the proportion of refills for which the waiting time was zero from 41 to $60 \%$ and then $81 \%$. We also noticed the balancing of waiting times between stations. However, the quality criterion was not met in the communication scenario for station 3 . The best remedy for this would be to add a charging point to this station, which was the most stressed one.
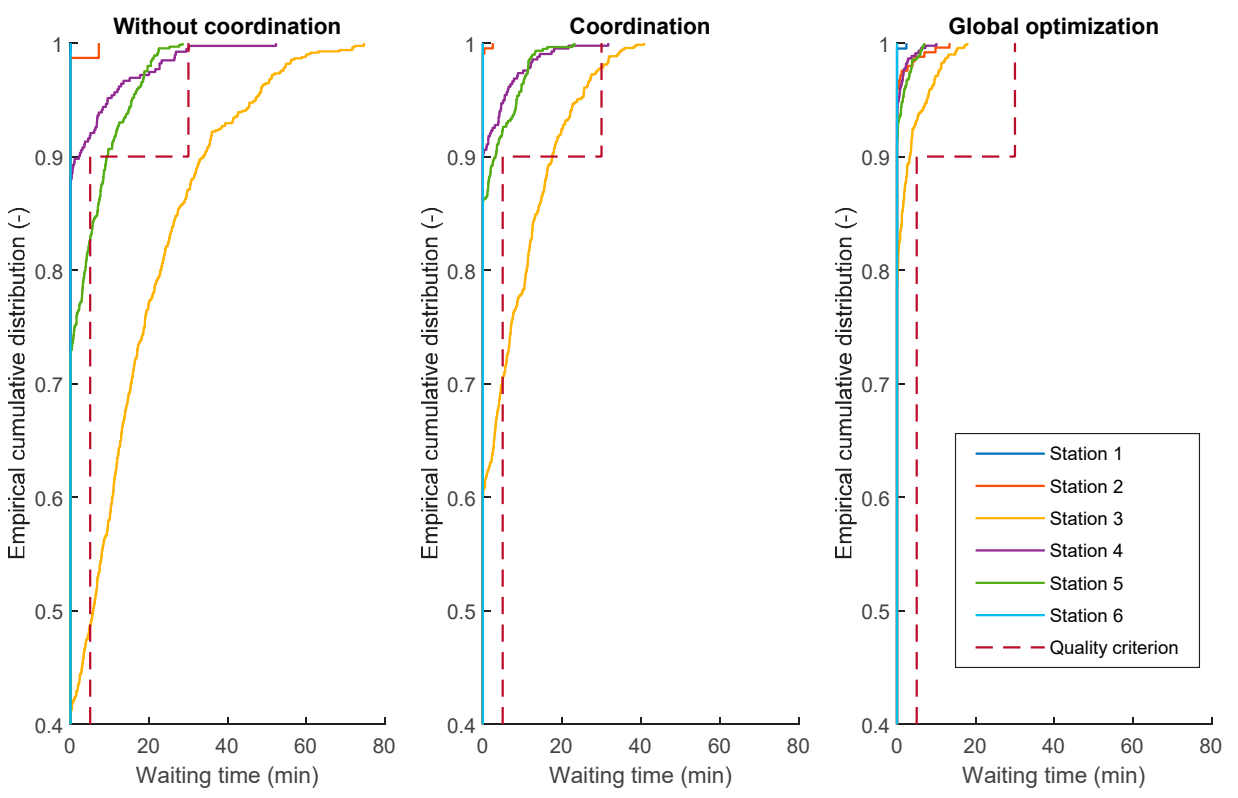

Figure 13. Waiting times at charging station, 3 charging points per station. 
Table 3 shows the average waiting and charging times for the three strategies. There was a slight increase in recharging time and a decrease in waiting times. This changeover showed the choices made by some people to recharge at lower power to reduce their waiting time (low power stations were less demanded, resulting in a lower waiting time). Thus, the implementation of the communication system could reduce total time spent in stations by $9 \%$ compared to the situation without communication, out of a potential decrease of $13 \%$ with overall optimization.

Table 3. Average charging and waiting time for the three scenarios.

\begin{tabular}{cccc}
\hline Results & No Coordination & Communication & Global Optimization \\
\hline Average charging time (min) & 46 & 47.3 & 47.7 \\
Average waiting time (min) & 15.6 & 8.8 & 5.8 \\
Total (min) & 61.6 & 56.1 & 53.5 \\
Saving compared to No C. & - & $9 \%$ & $13 \%$ \\
\hline
\end{tabular}

Figure 14 shows the same comparison in a more crowded situation. There was the same number of cars per day, but only two charging points per station. The decrease in the area above the curve was even more pronounced than in the previous situation, particularly for charging station number 3 . The time spent in stations was then reduced by respectively $46 \%$ and $55 \%$ with communication and overall optimization, compared to the uncoordinated scenario.
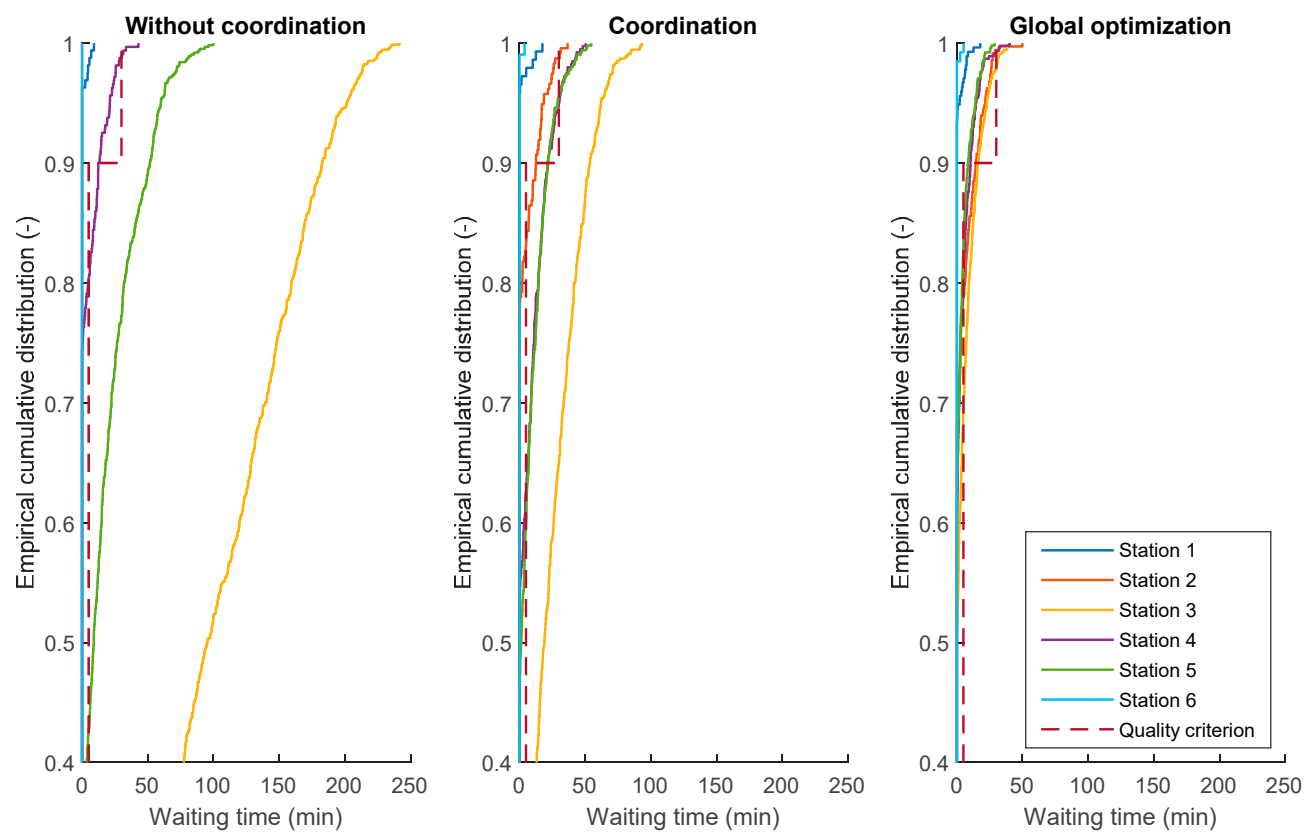

Figure 14. Waiting times at charging station, 2 charging points per station.

In conclusion, we noted two things about the performance of the proposed communication solution: the improved distribution of vehicles, reducing the time spent in stations to a level close to the ideal distribution, as well as the increased efficiency in the event of high traffic.

We could thus extrapolate the use of such a communication system in more complex situations, such as with several possible routes, traffic jams, variable speeds, and consumption. Those more advanced functions could be added without modifying the system's structure, but only by refining the calculation of charging plans. 


\section{Conclusions}

In conclusion, the research question of this article was to explore how a system of communication between EV and CS could reduce travel time during long-distance trips. We presented an adapted differential evolution algorithm. By adding two mutations, we improved the convergence speed. This algorithm enabled us to approach the optimal distribution of the recharge session of the traveling vehicles on a given day. We also presented a communication scheme between electric vehicles, which allowed them to coordinate and limit their waiting times at charging stations. We worked to make this system as easy to deploy as possible by minimizing communication needs and choosing a distributed architecture. The parametric study showed us that it was possible to achieve a good compromise between system performance and communication needs. Finally, a comparison of its performance against an uncoordinated situation and one with overall optimization showed a $9 \%$ gain in time spent in stations out of a potential of $13 \%$. In addition, the system was ever more useful in crowded situations, with a time gain of $46 \%$ out of a potential of $55 \%$. In the future, it is worth studying the robustness of such a system when not all travelers use it or follow recommendations, as well as the tradeoff between trip duration and cost, in the case where charging stations have different prices depending on their maximum power. This study also raised the question of the cost-benefit analysis of such a system.

Author Contributions: Conceptualization, all authors; methodology, all authors; software, J.H.; validation, all authors; formal analysis, all authors; resources, Z.D.; data curation, J.H.; writingoriginal draft preparation, J.H.; writing—review and editing, all authors; supervision, P.D., M.P. and Z.D. All authors have read and agreed to the published version of the manuscript.

Funding: This research benefits from the support of the ANRT association and Groupe PSA.

Conflicts of Interest: The authors declare no conflict of interest.

\section{Abbreviations and Variables}

EV
SoC
CS
$E_{j, n}$
$E_{\text {Required }, n}$
$N_{\text {ch }}$
SoC $C_{\text {Max }, n}$
SoC Min,n
SoC $C_{\text {exit }, n}$
SoC $C_{\text {exit required, } n}$
$T$
$T_{\text {adv }, n}$
$T_{\text {Trip }, n}$
$T_{\text {Driving }, n}$
$T_{\text {Station } j, n}$
$T_{\text {Wait } j, n}$
$T_{\text {Charge } j, n}$
$T_{\text {Other } j, n}$

Electric vehicle Battery state of charge (-) Charging station

Energy stored by EV $n$ at charging station $\mathrm{j}(\mathrm{kWh})$

Energy required by vehicle $n$ to complete it trip $(\mathrm{kWh})$

Number of changes of charging plans made by EVs (-)

Maximum SoC during a charging session of vehicle $n(-)$

Minimum SoC of vehicle $n(-)$

SoC of EV $n$ when leaving the highway (-)

Required SoC of EV $n$ when leaving the highway (-)

Refreshment period ( $\mathrm{min}$ ) of drivers requirements

For EV $n$, time between the beginning of its trip (at home) and its entrance on the highway (min)

Trip time (min) of EV $n$

Driving time ( $\mathrm{min})$

Time spent by EV $n$ in charging station $j$ (min)

Waiting time for an available charging point, when the station is full (min)

Time required to store the intended amount of energy ( $\mathrm{min}$ )

Duration needed for all other operations in a charging station: decelerating, accessing the station, launching the charging session (min)

\section{References}

1. IPCC; Masson-Delmotte, V.; Zhai, P.; Pörtner, H.-O.; Roberts, D.; Skea, J.; Shukla, P.; Pirani, A.; Moufouma-Okia, W.; Péan, C.; et al. Global Warming of $1.5^{\circ} \mathrm{C}$. An IPCC Special Report on the Impacts of Global Warming of $1.5^{\circ} \mathrm{C}$ above Pre-Industrial Levels and Related Global Greenhouse Gas Emission Pathways, in the Context of Strengthening the Global Response to the Threat of Climate Change, Sustainable Development, and Efforts to Eradicate Poverty. 2018. Available online: https://www.ipcc.ch/sr15/ (accessed on 11 May 2021). 
2. Solaymani, S. $\mathrm{CO}_{2}$ Emissions Patterns in 7 Top Carbon Emitter Economies: The Case of Transport Sector. Energy 2019, 168, 989-1001. [CrossRef]

3. Moro, A.; Lonza, L. Electricity Carbon Intensity in European Member States_Impacts on GHG Emissions of Electric Vehicles. Transp. Res. Part D Transp. Environ. 2018, 64, 5-14. [CrossRef] [PubMed]

4. Faria, R.; Marques, P.; Moura, P.; Freire, F.; Delgado, J.; de Almeida, A.T. Impact of the Electricity Mix and Use Profile in the Life-Cycle Assessment of Electric Vehicles. Renew. Sustain. Energy Rev. 2013, 24, 271-287. [CrossRef]

5. Singh, B.; Strømman, A.H. Environmental Assessment of Electrification of Road Transport in Norway: Scenarios and Impacts. Transp. Res. Part D Transp. Environ. 2013, 25, 106-111. [CrossRef]

6. Borne, O.; Perez, Y.; Petit, M. Market Integration or Bids Granularity to Enhance Flexibility Provision by Batteries of Electric Vehicles. Energy Policy 2018, 119, 140-148. [CrossRef]

7. Varga, B.O.; Sagoian, A.; Mariasiu, F. Prediction of Electric Vehicle Range: A Comprehensive Review of Current Issues and Challenges. Energies 2019, 12, 946. [CrossRef]

8. Donkers, A.; Yang, D.; Viktorović, M. Influence of Driving Style, Infrastructure, Weather and Traffic on Electric Vehicle Performance. Transp. Res. Part D Transp. Environ. 2020, 88, 102569. [CrossRef]

9. Csonka, B.; Csiszár, C. Determination of Charging Infrastructure Location for Electric Vehicles. Transp. Res. Procedia 2017, 27, 768-775. [CrossRef]

10. Funke, S.Á.; Plötz, P.; Wietschel, M. Invest in Fast-Charging Infrastructure or in Longer Battery Ranges? A Cost-Efficiency Comparison for Germany. Appl. Energy 2019, 235, 888-899. [CrossRef]

11. Kosmanos, D.; Maglaras, L.A.; Mavrovouniotis, M.; Moschoyiannis, S.; Argyriou, A.; Maglaras, A.; Janicke, H. Route Optimization of Electric Vehicles Based on Dynamic Wireless Charging. IEEE Access 2018, 6, 42551-42565. [CrossRef]

12. del Razo, V.; Jacobsen, H.-A. Smart Charging Schedules for Highway Travel With Electric Vehicles. IEEE Trans. Transp. Electrif. 2016, 2, 160-173. [CrossRef]

13. Gusrialdi, A.; Qu, Z.; Simaan, M.A. Distributed Scheduling and Cooperative Control for Charging of Electric Vehicles at Highway Service Stations. IEEE Trans. Intell. Transp. Syst. 2017, 18, 2713-2727. [CrossRef]

14. Qin, H.; Zhang, W. Charging Scheduling with Minimal Waiting in a Network of Electric Vehicles and Charging Stations. In Proceedings of the Eighth ACM International Workshop on Vehicular Inter-Networking, Las Vegas, NV, USA, 19-23 September 2011; pp. 51-60.

15. Tan, J.; Wang, L. Real-Time Charging Navigation of Electric Vehicles to Fast Charging Stations: A Hierarchical Game Approach. IEEE Trans. Smart Grid 2015, 8, 846-856. [CrossRef]

16. Yang, Y.; Yao, E.; Yang, Z.; Zhang, R. Modeling the Charging and Route Choice Behavior of BEV Drivers. Transp. Res. Part $C$ Emerg. Technol. 2016, 65, 190-204. [CrossRef]

17. Hassler, J.; Dimitrova, Z.; Petit, M.; Dessante, P. Service for Optimization of Charging Stations Selection for Electric Vehicles Users during Long Distances Drives: Time-Cost Tradeoff. IOP Conf. Ser. Mater. Sci. Eng. 2020, 1002, 012029. [CrossRef]

18. IEC 62196-3:2014 I IEC Webstore I LVDC. Available online: https:/ / webstore.iec.ch/publication/6584\&preview=1 (accessed on 23 January 2020).

19. Salapic, V.; Grzanic, M.; Capuder, T. Optimal Sizing of Battery Storage Units Integrated into Fast Charging EV Stations. In Proceedings of the 2018 IEEE International Energy Conference (ENERGYCON), Limassol, Cyprus, 3-7 June 2018 ; pp. 1-6.

20. Price, K.; Storn, R.M.; Lampinen, J.A. Differential Evolution: A Practical Approach to Global Optimization; Springer Science \& Business Media: Berlin/Heidelberg, Germany, 2006; ISBN 978-3-540-31306-9.

21. DiRIF Les comptages en Ile-de-France-Direction des routes Ile-de-France. Available online: http://www.dir.ile-de-france. developpement-durable.gouv.fr/les-comptages-a174.html (accessed on 13 March 2019).

22. Data.gouv.fr Trafic Moyen Journalier Annuel sur le Réseau Routier National. Available online: /fr/datasets/trafic-moyenjournalier-annuel-sur-le-reseau-routier-national/ (accessed on 22 January 2020). 